\title{
Polarized Positive and Negative Muon Beams to perform DVCS Measurements at COMPASS
}

\author{
Nicole d'Hose \\ CEA-Saclay, Irfu/SphN, 91191 Gif-sur-Yvette, France \\ on behalf of the COMPASS Collaboration
}

\begin{abstract}
The high energies available at CERN, and the option of using either positive or negative polarized muon beams, make the fixed-target COMPASS set-up a unique place for studying GPDs, through Deeply Virtual Compton Scattering (DVCS). A GPD program is part of the Medium and Long Term Plans at COMPASS [1]. This contribution presents the methodology and the goal of such experiments.
\end{abstract}

Keywords: Nucleon Structure, Generalized Parton Distributions, Virtual Compton scattering PACS: $13.60 . \mathrm{Fz} ; 13.60 . \mathrm{Hb} ; 13.85 . \mathrm{Hd} ; 14.20 . \mathrm{Dh} ; 14.65 .-\mathrm{q} ; 25.30 . \mathrm{Mr} ; 24.85 .+\mathrm{p}$

\section{HIGH ENERGY MUON BEAM AND KINEMATIC DOMAIN COVERED AT COMPASS}

The COMPASS apparatus is located on the unique high-energy (200 GeV) and highly polarized $\mu^{ \pm}$beam lines of the CERN SPS and uses a high resolution forward spectrometer in conjunction with a fixed target (unpolarized or longitudinally or transversely polarized). By installing a recoil proton detector around the target to ensure exclusivity of Deeply Virtual Compton Scattering (DVCS) and Deeply Virtual Meson Production (DVMP) events, it could be converted into a facility measuring exclusive reactions within a kinematic subspace ranging from $x \sim 0.01$ to $\sim 0.1$, which cannot be explored by any other existing or planned facility in the near future. Fig. 1 displays the kinematic domain of fixed-

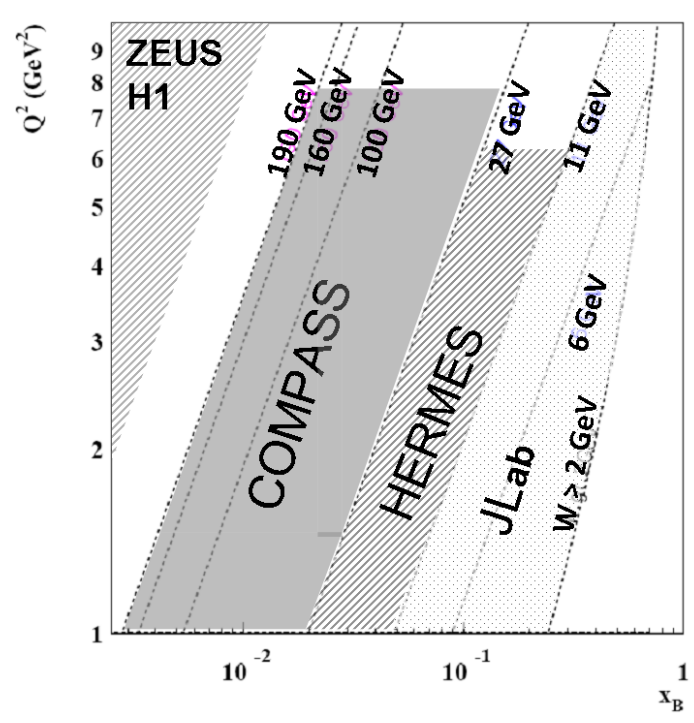

FIGURE 1. Kinematic domain of all the world experiments. target experiments COMPASS, HERMES and JLab. These domains are indicated between the dotted lines defined by the maximum energy of the facility and the curve $\mathrm{W}$ $>2 \mathrm{GeV}$, i.e. above the resonance domain. The kinematic limit of the HERA collider experiments $\mathrm{H} 1$ and ZEUS, investigating the very small $x$ domain, is also displayed. COMPASS would thus explore the uncharted $x$ domain between those of $\mathrm{H} 1$ and ZEUS at the HERA collider and of fixed-target experiments as HERMES and the planned 12 $\mathrm{GeV}$ extension of the JLab accelerator. 


\section{METHODOLOGY WITH HIGH ENERGY POLARIZED $\mu^{+}$AND $\mu^{-}$BEAMS AND DVCS MEASUREMENTS}

DVCS is considered to be the theoretically cleanest of the experimentally accessible processes because effects of next-to-leading order and subleading twist are under theoretical control [2]. The competing Bethe-Heitler $(\mathrm{BH})$ process which is elastic lepton-nucleon scattering with a hard photon emitted by either the incoming or outgoing lepton, has a final state identical to that of DVCS so that both processes interfere on the level of amplitudes $\mathscr{A}$ :

$$
d \sigma(\mu \mathrm{N} \rightarrow \mu \mathrm{N} \gamma) \propto\left|\mathscr{A}_{B H}\right|^{2}+\left|\mathscr{A}_{D V C S}\right|^{2}+\underbrace{\mathscr{A}_{B H} \mathscr{A}_{D V C S}^{*}+\mathscr{A}_{B H}^{*} \mathscr{A}_{D V C S}}_{I}
$$

COMPASS offers the advantage to provide various kinematic domains where either BH or DVCS dominates (see Fig. 2). The Bethe-Heitler amplitude is well known (it relies only on the knowledge of elastic nucleon form factors). The collection of almost pure $\mathrm{BH}$ events at small $x$ allows one to get an excellent reference yield and to control accurately the global efficiency of the apparatus. In contrast the collection of almost pure DVCS events at larger $x$ will allow the measurement of the $x$-dependence of the $\mathrm{t}$-slope of the cross section which is related to the tomographic partonic image of the nucleon. In the intermediate domain, the DVCS contribution will be boosted by the $\mathrm{BH}$ process through the interference term. COMPASS is presently the only facility to provide polarized leptons with either charge: polarized $\mu^{+}$and $\mu^{-}$beams. As the $\mathrm{BH}$ is independent of charge and polarization, this contribution can be removed by subtracting 2 separate measurements obtained for the two beam charges. Moreover the natural polarization of the muon beam produced from pion decay changes sign when the beam charge is reversed and the different topologies of $\mu^{+}$and $\mu^{-}$, polarized with opposite direction, allow one to select only the real part or the imaginary part of the complex amplitude of DVCS.
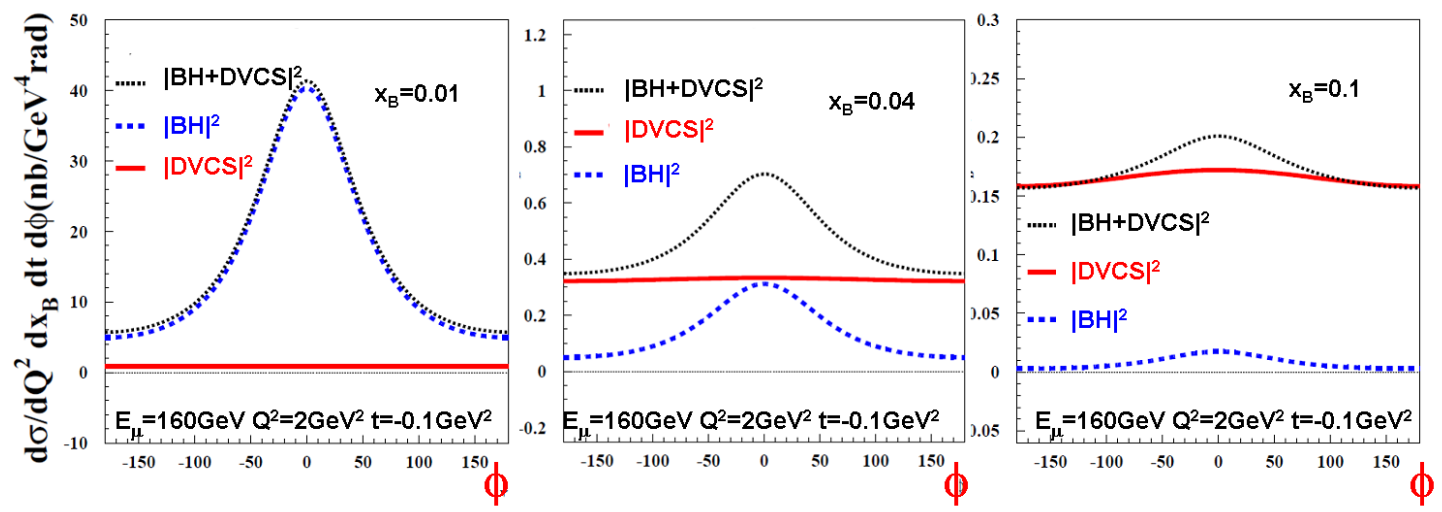

FIGURE 2. $\mathrm{BH}$ (dashed line), DVCS (solid line) and $\mu p \rightarrow \mu^{\prime} p \gamma$ total single photon production (dotted line) contributions for $\mathrm{x}=0.01,0.04$ and 0.1 at $Q^{2}=2 \mathrm{GeV}^{2},|t|=0.1 \mathrm{GeV}^{2}$ and $E_{\mu}=160 \mathrm{GeV}$. 
For the muo-production of real photons off an unpolarized proton target, the differential cross section can be written as ${ }^{1}$ :

$$
\frac{d^{4} \sigma(\mu p \rightarrow \mu p \gamma)}{d x_{B} d Q^{2} d|t| d \phi}=d \sigma^{B H}+\left[d \sigma_{\text {unpol }}^{D V C S}+P_{\mu} d \sigma_{\text {pol }}^{D V S C}\right]+e_{\mu}\left[\operatorname{Re} I+P_{\mu} \operatorname{Im} I\right]
$$

where $I$ is the interference term of Eq. (1), $P_{\mu}$ is the beam polarization and $e_{\mu}$ its charge in units of the elementary charge. The DVCS amplitude can be expanded in $1 / Q$ beyond leading twist- 2 including all twist-3 contributions [2]. The dependence on $\phi$, the azimuthal angle between lepton scattering plane and photon production plane, is a characteristic feature of the cross section. Integration over $\phi$ and/or analysis of the angular dependence in $\phi$ allows us to isolate specific contributions that are sensitive to different combinations of quark GPDs. Gluon GPDs enter in DVCS only beyond leading order in $\alpha_{s}(\mathrm{LO})$, analogous to DIS.

At the CERN SPS M2 beamline, the 'natural' polarization of the muon beam produced from pion decay changes sign when the beam charge is reversed. Hence with the same apparatus the COMPASS experiment can perform separate measurements for the two beam charge/polarization states $\leftleftarrows$ and $\rightarrow$, which can be used to calculate:

the 'Beam Charge $(C)$ and Spin $(S)$ Difference' (for Unpolarized $(U)$ proton target)

$$
\begin{aligned}
\mathscr{D}_{U, C S} & \equiv d \sigma^{\longleftarrow}-d \sigma^{\rightrightarrows}=2\left[P_{\mu} d \sigma_{\text {pol }}^{D V C S}+e_{\mu} \operatorname{Re} I\right] \\
& \propto\left(\left\{s_{1}^{D V C S} \sin \phi\right\}\right)+\left(c_{0}^{I}+c_{1}^{I} \cos \phi+\left\{c_{2}^{I} \cos 2 \phi+c_{3}^{I} \cos 3 \phi\right\}\right)
\end{aligned}
$$

in which the BH contribution cancels out. The coefficients $c_{n}^{D V C S}$ and $s_{n}^{I}$ are related to certain combinations of Compton Form Factors (CFFs). A CFF $\mathscr{F}$ is a sum over flavors $f$, of convolutions of the respective GPDs $F^{f}$ with a perturbatively calculable kernel describing the hard $\gamma^{*} q$ interaction. Note that each contribution in Eq. 3 and Eq. 4 shown between a pair of braces corresponds to higher-twist or higher-order effects. The analysis of the $\phi$-dependence of the beam charge and spin difference $\mathscr{D}_{U, C S}$ will provide via the term $\operatorname{Re} I$ the two leading twist-2 expansion coefficients $c_{0}^{I}$ and $c_{1}^{I}$, the dominant contribution to which is related to the real part of the Compton form factor $\mathscr{H}$ that is in LO given by a flavor sum of convolutions involving the GPDs $\mathrm{H}^{f}$.

The 'Beam Charge and Spin Sum' of cross sections can also be evaluated:

$$
\begin{aligned}
\mathscr{S}_{U, C S} & \equiv d \sigma^{\stackrel{+}{ }}+d \sigma^{\bar{\rightrightarrows}}=2\left[d \sigma^{B H}+d \sigma_{\text {unpol }}^{D V C S}+e_{\mu} P_{\mu} \operatorname{Im} I\right] \\
& \propto 2\left[d \sigma^{B H}\right]+\left(c_{0}^{D V C S}+\left\{c_{1}^{D V C S} \cos \phi+c_{2}^{D V C S} \cos 2 \phi\right\}\right)+\left(s_{1}^{I} \sin \phi+\left\{s_{2}^{I} \sin 2 \phi\right\}\right)
\end{aligned}
$$

in which the $\mathrm{BH}$ contribution does not cancel out. 1) The analysis of the $\phi$-dependence of the beam charge\&spin sum $\mathscr{S}_{U, C S}$ will provide via the term Im $I$ the leading twist-2 quantity $s_{1}^{I}$. Its dominant contribution is related to the imaginary part of the Compton form factor $\mathscr{H}$. 2) A parallel analysis can be performed subtracting the BH contribution

\footnotetext{
${ }^{1}$ For simplicity $d \sigma$ is used in the following instead of $\frac{d^{4} \sigma(\mu p \rightarrow \mu p \gamma)}{d x_{B} d Q^{2} d|t| d \phi}$
} 
when it is not too large, and integrating over $\phi$ to get rid of the complete interference term and of the $\phi$-dependent terms of the DVCS contribution. Thus the DVCS leading twist-2 quantity $c_{0}^{D V C S}$ can be isolated and its characteristic $t$-slope can be determined as a function of $x$, from which conclusions can be drawn on the transverse size of the nucleon over the $x$-range accessible to COMPASS ('nucleon tomography').

\section{$x$-DEPENDENCE OF THE $t$-SLOPE OF THE DVCS CROSS SECTION RELATED TO NUCLEON TOMOGRAPHY}

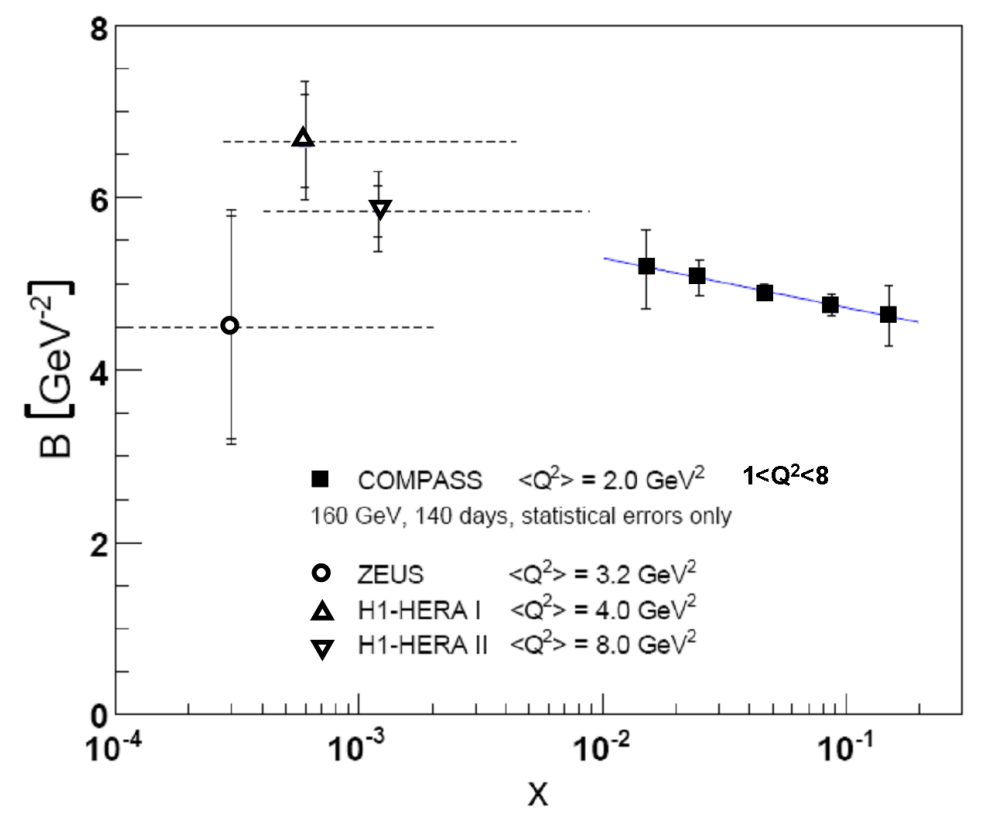

FIGURE 3. The $x$ dependence of the fitted $t$-slope parameter $B$ of the DVCS cross section, expressed as $d \sigma / d t \propto e^{-B|t|}$. COMPASS projections are calculated for $1<Q^{2}<8 \mathrm{GeV}^{2}$ and are compared to some HERA results for which the mean value $<Q^{2}>$ is in this range. Projections are done for 140 days of running time with a $2.5 \mathrm{~m} \mathrm{LH}_{2}$ target, an intensity of $4.6 \times 10^{8} \mu$ in a $48 \mathrm{~s}$ SPS spill period and an overall global efficiency $\varepsilon_{\text {global }}=0.1$

Using the $\phi$-integrated beam charge and spin sum (Eq.5) after BH subtraction, Fig. 3 shows the projected statistical accuracy for a measurement at COMPASS of the $x$-dependence of the $t$-slope parameter $B(x)$ of the DVCS cross section $\frac{d \sigma}{d t}(x) \propto$ $\exp (-B(x)|t|)$. In the simple ansatz $B(x)=B_{0}+2 \alpha^{\prime} \log \left(\frac{x_{0}}{x}\right)$, the shrinkage parameter $\alpha^{\prime}$ is known a long time to describe the decrease in nucleon size with increasing $x$. More recently, this ansatz was also used for the 'reggeized' description of a correlated $x, t$ dependence of GPDs. The $t$-slope of the GPD $H^{f}$ at a given $x$ was shown [3] to be related to the average impact parameter $\left\langle\left(b_{\perp}^{f}\right)^{2}\right\rangle$ in the distribution of partons of flavor $f$ carrying the longitudinal momentum fraction $x$ at a given $t: B^{f}(x) \sim 1 / 2\left\langle\left(b_{\perp}^{f}\right)^{2}\right\rangle(x)$.

Data on $B$ exist only for the HERA collider $x$-range from $10^{-4}$ to $0.01[5,6]$, below the COMPASS range $0.01<x<0.1$. In Fig. 3 only HERA results are reported for which the mean value $\left\langle Q^{2}\right\rangle$ is in the investigated domain by COMPASS. In the valence region, 
where no experimental determinations of $B$ exist, some information comes from fits adjusted to form factor data which give $\alpha^{\prime} \simeq 1 \mathrm{GeV}^{2}$ [7, 8]. For the low- $x$ sector, H1 results on $\alpha^{\prime}$ from exclusive $J / \psi$ production [9], which involves the generalized gluon distribution, are smaller by two standard deviations in the total experimental uncertainty than the corresponding value $\alpha^{\prime}=0.25$ for Pomeron exchange in soft scattering processes. For the simulation shown in Fig. 3 we chose the values $\alpha^{\prime}=0.125$.

This measurement will yield new and significant information in the context of 'nucleon tomography' as it is expected in chiral-dynamics approach [4] and reported in Fig. 4. In this approach, the gluon density is generated by the 'pion cloud' of the nucleon so that a significant increase in the transverse size of the nucleon is predicted for $x$ below the ratio of pion and proton masses, $m_{\pi} / m_{p} \approx 0.15$ (this is in the investigated domain by COMPASS).

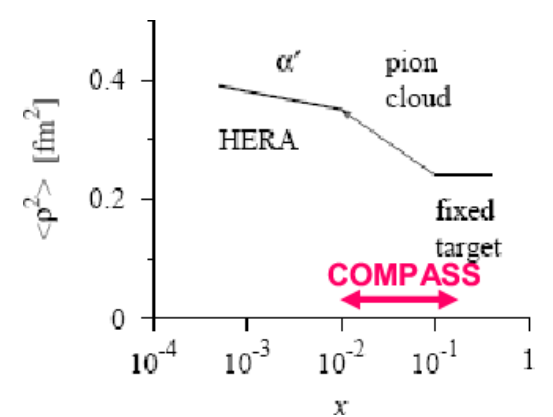

FIGURE 4. The average squared transverse radius of the gluon distribution in the nucleon (extracted from $J / \psi$ photoproduction data at HERA) and the COMPASS kinematic domain.

\section{MEASUREMENT OF THE BEAM CHARGE AND SPIN ASYMMETRY AND COMPARISON TO MODELS}

Using the beam charge and spin asymmetry $\mathscr{D}_{U, C S} / \mathscr{S}_{U, C S}$, Fig. 4 shows the projected statistical accuracy in a particular $\left(x, Q^{2}\right)$ bin, for a measurement of its $\phi$-dependence. Two of the curves are calculated using the 'VGG' GPD model [10]. As this model is meant to be applied mostly in the valence region, typically the value $\alpha^{\prime}=0.8$ is used in the 'reggeized' parameterization of the correlated $x, t$ dependence of GPDs. For comparison, also the model result for the 'factorized' $x, t$ dependence is shown, which corresponds to $\alpha^{\prime} \approx 0.1$ in the 'reggeized' ansatz.

A recent theoretical development exploits dispersion relations for Compton form factors. In this context, the additional curve is the result of a fitting procedure [11] including next-to-next-to leading order (NNLO) corrections which was developed and successfully applied to describe DVCS observables from very small values of $x$, for the HERA collider to large $x$ for HERMES and JLab.

\section{PLANS FOR A GPD PROGRAM AT COMPASS}

In the full-scale programme, two subsequent running periods of one year each are proposed, the first one for which the methodology has been described using an upolarized proton target, the second one using a transversely polarized proton target, in order to collect data that constrain mostly the GPDs $H$ and $E$.

Already a first observation of single-photon production in the COMPASS apparatus was successfully achieved in autumn 2008 . This will be followed up by a more quantitative test during 2 weeks in autumn 2009. 


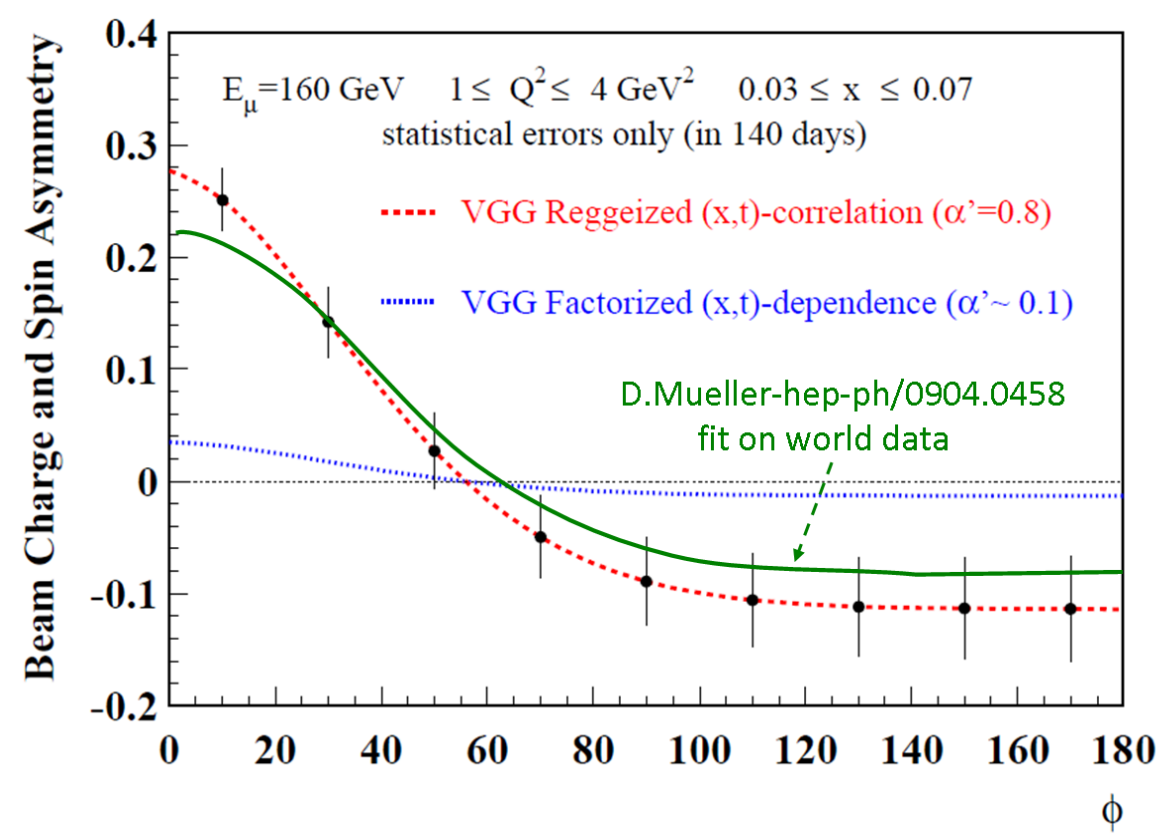

FIGURE 4. Projections for the Beam Charge and Spin Asymmetry measured at COMPASS for $0.03 \leq x \leq 0.07$ and $1 \leq Q^{2} \leq 4 \mathrm{GeV}^{2}$ and for 140 days of running time with a $2.5 \mathrm{~m} \mathrm{LH}_{2}$ target, an intensity of $4.6 \times 10^{8} \mu$ in a 48 s SPS spill period and an overall global efficiency $\varepsilon_{\text {global }}=0.1$. The dashed and dotted curves correspond to different variants of the VGG model [10] while the solid curve shows predictions based on the first fit on world data [11].

\section{REFERENCES}

1. COMPASS Medium and Long Term Plans, The COMPASS Collaboration, CERN-SPSC-2009-003, SPSC-I-238, January 21, 2009.

2. A.V. Belitsky, D. Müller and A. Kirchner, Nucl. Phys. B 629 (2002) 323.

3. M. Burkardt, Phys. Rev. D 62 (2000) 071503; erratum-ibid. d 66 (2002) 119903; Int. J. Mod. Phys. A 18 (2003) 173; Phys. Lett. B 595 (2004) 245.

4. M. Strikman and C. Weiss, Phys. Rev. D69 (2004) 054012.

5. H1, A. Aktas et al., Eur. Phys. J.C 44 (2005) 1, F.D. Aaron et al., Phys. Lett. B 659 (2008) 796.

6. ZEUS, S. Chekanov et al., DESY-08-178, arXiv:hep-exp:0812.2517v3.

7. M. Diehl, Th. Feldmann, R. Jakob and P. Kroll, Eur. Phys. J. C 39 (2005) 1.

8. M. Guidal, M.V. Polyakov, A.V. Radyushkin and M. Vanderhaeghen, Phys. Rev. D 72 (2005) 054013.

9. H1, A. Aktas et al., Eur. Phys. J.C 46 (2006) 585.

10. M. Vanderhaeghen, P.A.M. Guichon and M. Guidal, Phys. Rev. Lett. 80 (1998) 5064; Phys. Rev. D 60 (1999) 094017; K. Goeke, M.V. Polyakov and M. Vanderhaeghen, Prog. Part. Nucl. Phys. 47 (2001) 401.

11. K. Kumericki,D. Mueller and K. Passek-Kumericki, Nucl. Phys. B 794 (2008) 244, K. Kumericki and D. Mueller, arXiv 0904.0458[hep-ph] 